\title{
The Medical Imaging \& Technology Alliance Conference on Research Endpoints Appropriate for Medicare Coverage of New PET Radiopharmaceuticals
}

\author{
Bruce J. Hillman, MD*1,2, Richard A. Frank, MD, $\mathrm{PhD}^{3}$, and Brian C. Abraham, MPA ${ }^{4}$ \\ ${ }^{I}$ Department of Radiology and Medical Imaging and the Department of Public Health Sciences, University of Virginia, \\ Charlottesville, Virginia; ${ }^{2}$ ACR Image Metrix, Philadelphia, Pennsylvania; ${ }^{3}$ Frank Healthcare Advisers, PC, Alexandria, Virginia; \\ and ${ }^{4}$ Medical Imaging \& Technology Alliance, Arlington, Virginia
}

The outcomes of a 2011 Medical Imaging \& Technology Alliance (MITA) conference helped shape considerations about what might be the most appropriate pathways for the regulatory and payment considerations of new PET radiopharmaceuticals. As follow-up to that conference, MITA convened a second conference of stakeholders to advise payers on what might be acceptable endpoints for clinical trials to support the coverage of novel PET agents. The conference involved experts on imaging and clinical research, providers of PET services, as well as representatives of interested medical societies, the PET industry, and the regulatory and payer communities. The principal outcome of their deliberations was that it was unrealistic to expect trials of new PET radiopharmaceuticals to directly demonstrate a health benefit. Rather, intermediate outcomes, such as a positive change in patient management, would be more efficient and appropriate.

Key Words: PET; National Oncologic PET Registry; regulation; payment; clinical trial endpoints; diagnostic endpoints

J Nucl Med 2013; 54:1675-1679

DOI: $10.2967 /$ jnumed.113.127886

\section{INTRODUCTION}

A 2011 Medical Imaging \& Technology Alliance (MITA) conference addressed appropriate pathways for new PET radiopharmaceuticals to be considered for Medicare coverage by CMS (1). The outcomes of that conference helped shape CMS' decision to reconsider its current National Coverage Determination (NCD) for PET, which precludes reimbursement for any PET procedures that are not covered under the NCD (or via a coverage with evidence development [CED] program); this current policy effectively requires that new PET radiopharmaceuticals must undergo the scrutiny of a national coverage analysis. Recently, CMS proposed a new pathway for coverage of novel oncologic PET procedures that would allow local Medicare Administrative Contractors to decide whether or not to reimburse for these procedures (2).

Corresponding author and reprints: Bruce J. Hillman, MD, 13129 Adona Lane, Wake Forest, NC 27587.

E-mail: BJH8A@virginia.edu

${ }^{*} \mathrm{Dr}$. Hillman is a consultant to and Chief Scientific Officer of ACR Image Metrix, an imaging contract research organization owned by the ACR.

Published online Aug. 1, 2013.

COPYRIGHT (C) 2013 by the American College of Radiology. Printed with permission from the American College of Radiology.
In November 2012, MITA picked up the thread of the 2011 conference by convening a second conference of experts and stakeholders. Given that the use of evidence-based criteria to determine the utility of PET procedures has been a hallmark of the information MITA has collected and conveyed to interested stakeholders, the 2012 conference addressed which possible clinical research endpoints are appropriate evidentiary standards to demonstrate that new PET radiopharmaceuticals and new PET procedures lead to improved outcomes for Medicare beneficiaries, and thus, should be covered.

The conference involved experts on imaging, clinical research, and providers of PET services, as well as representatives of interested medical societies, the PET industry, and the regulatory and payer communities. Those attending participated in lectures, small group breakout sessions, and consensus-building that led to a convergence of opinion on clinical endpoints suitable for evaluation of new PET procedures.

From the outset, there was general agreement on the following key issues laid forth by Louis Jacques, MD, head of the CMS Coverage and Analysis Group:

- In and of themselves, diagnostic tests, such as imaging examinations, do not have a direct therapeutic effect. The potential benefits of diagnostic tests relate to their providing information to optimize treatment plans and, thereby, improve clinical care and health outcomes.

- The potential benefits and harms associated with diagnostic tests accrue from the capacity of the test to inform downstream clinical management of the patient.

- The balance of risk and harm, the acuity and severity of the patient's condition, characteristics of the test, and the likelihood of a positive result should inform a caregiver's decision to perform a test.

- Diagnostic tests can help avoid futile treatment; by so doing, they can improve health outcomes.

The goal of the Medicare coverage determination process is to assess the value of using a new medical technology or a new application of an existing technology in the care of patients. For many therapeutic technologies, the approach can be quite straightforward. Trials of therapeutic innovations usually can be designed to directly demonstrate that using the technology improves important health outcomes, such as lengthening survival relative to the current standard of care or doing nothing at all.

Advanced diagnostic imaging technologies, such as PET, are different. A diagnostic test is nearly always embedded in a chain of other diagnostic tests and therapeutic interventions that constitute 


\begin{tabular}{ll}
\hline \multicolumn{1}{c}{ More persuasive } & \multicolumn{1}{c}{ Less persuasive } \\
\hline $\begin{array}{c}\text { Longer life and improved function or participation in } \\
\text { critical activities }\end{array}$ & $\begin{array}{l}\text { Longer life with declining function or participation in } \\
\text { critical activities } \\
\text { Longer life with arrested decline }\end{array}$ \\
$\begin{array}{l}\text { Improved disease-specific survival without improved } \\
\text { overall survival }\end{array}$ \\
$\begin{array}{l}\text { Significant symptom improvement allowing better } \\
\text { function or participation in critical activities }\end{array}$ & Surrogate test result is improved \\
Reduced need for burdensome tests and treatments & Test produces a better image \\
Improved quality of life & Improves physician confidence \\
\hline${ }^{*}$ Adapted from Dr Louis Jacques' presentation at the MITA conference, November 2012.
\end{tabular}

clinical care. Separating out the specific contribution to health of the test from all other care is almost always too expensive, too time consuming, and confounded by the multifactorial nature of patient management. Hence, directly measuring the effect of employing imaging is impractical for all but the most critical applications. ${ }^{1}$ That being the case, Dr Jacques proposed an approach that formed the basis for subsequent deliberations (Verbal communication, Louis Jacques, MD, lecture entitled "Medicare and Diagnostic Imaging: Engaging on Evidence and Outcomes," MITA PET Endpoints Workshop, November 13, 2012). For a new PET radiopharmaceutical to achieve Medicare coverage, the sponsor should provide adequate evidence that, compared to alternatives, the incremental information obtained using the new PET technology changes physician recommendations, resulting in changes in therapy that may improve clinically meaningful health outcomes in Medicare beneficiaries. He went on to posit health outcomes that CMS would view as persuasive, given rigorous data, and others the agency would find to be less so (Table 1).

For many new imaging technologies, the accumulation of iteratively derived data at clinical sites is substantial enough to support conventional local coverage decisions. However, particularly for innovations such as new PET radiopharmaceuticals—which proffer broad-based and far-reaching implications for care-extant data are often insufficient to support a coverage decision. In such cases in the past, the agency has determined that an NCD employing CED is the most efficient way to generate the necessary data while not unnecessarily restricting patient access to the benefits of this innovation. CED allows per-case payments to providers using the technology as part of a deemed trial or registry so long as they submit their data for analyses to guide CMS' coverage decision making. As a prime example, in 2004, CMS indicated its willingness to provide coverage under a CED program for performing 18-fluoro-deoxyglucose-PET exams on patients with cancers for which prior data were considered insufficient to support coverage. The National Oncologic PET Registry (NOPR), sponsored by the Academy of Molecular Imaging (now the World Molecular Imaging Society), and developed and managed by the American College of Radiology Imaging Network ${ }^{\circledR}$, analyzed the impact of

${ }^{1}$ As one example, the National Lung Screening Trial, a randomized clinical trial of annual chest x-ray versus annual low-dose CT screening for lung cancer, required nearly 10 years and more than $\$ 200$ million to demonstrate a $20 \%$ reduction in lung cancer mortality with CT. It is impossible to devote such resources to all but the most prevalent and deadly conditions.
PET on physicians' therapeutic plans for several hundred thousand patients (3-6). The clinical evidence gained via the NOPR contributed to a well-informed expansion of Medicare coverage for PET in cancer patients.

\section{RESEARCH DESIGNS AND ENDPOINTS APPLICABLE TO PET}

The technology assessment hierarchy (Table 2) for diagnostic tests, reviewed by Constantine Gatsonis, $\mathrm{PhD}$, using examples from the literature and ongoing trials, is applicable to the useful assessment of PET radiopharmaceuticals by both the FDA and CMS.

The FDA's evaluation of sponsor-submitted clinical evidence and subsequent approval to label and market the innovation in the United States precedes CMS coverage assessment. For new drugs, including contrast materials and radiopharmaceuticals, the FDA requires the sponsor to prove the drug is "safe and effective" for its intended use, whereas the CMS standard for coverage is that the medical service provided is "reasonable and necessary." The distinction may seem minor. However, the difference between the 2 benchmarks has implications for what constitutes sufficient proof to pass agency scrutiny. In contrast, CMS places its emphasis on a demonstration of incremental benefits in the health of a patient. As noted above, because of the difficulty in directly measuring the impact of diagnostic tests on health outcomes, it may be more suitable to design trials with intermediate outcomes that can be reasonably linked to improvements in health, rather than on a definitive health outcome, such as a measurable reduction in diseasespecific mortality. The central problem with this approach is that the association between an intermediate outcome and a definitive health outcome may or may not be well established. Alternatively, as noted by Dr Gatsonis and William Lawrence, MD, MS, of the Agency for Healthcare Research and Quality, it may be necessary to employ decision analytic methods based on modeling of clinical situations to establish the relationship. These outcomes should be appropriate to the intended use of a test; for example, to resolve a diagnostic dilemma, stage the disease for optimal choice of therapy, or to determine when treatment would be futile and therefore unjustifiably costly or injurious to the patient.

The NOPR is a prime example of the effectiveness of employing a relevant intermediate endpoint. As the name implies, the NOPR is an observational registry, rather than a randomized clinical trial in which subjects are randomly assigned to different arms. Thus, the results may better reflect actual use in clinical practice across a broad array of settings relevant to the Medicare population. All NOPR subjects received PET imaging for 
TABLE 2

The Diagnostic Imaging Technology Assessment Hierarchy*

\begin{tabular}{cc}
\hline Level of evaluation & Example measures \\
\hline Technical factors & $\begin{array}{c}\text { Spatial resolution, contrast } \\
\text { resolution, etc. } \\
\text { Case studies and } \\
\text { observational series }\end{array}$ \\
$\begin{array}{c}\text { Descriptive findings } \\
\text { Diagnostic efficacy }\end{array}$ & $\begin{array}{c}\text { Sensitivity, specificity, } \\
\text { ROC analysis, etc. }\end{array}$ \\
\hline Diagnostic impact & $\begin{array}{c}\text { Change in diagnostic } \\
\text { probabilities }\end{array}$ \\
Therapeutic impact & Change in treatment \\
Health outcomes & $\begin{array}{c}\text { Physical, mental, quality } \\
\text { of life changes }\end{array}$ \\
Cost-effectiveness & Cost/quality-adjusted year \\
& of life saved
\end{tabular}

\footnotetext{
*Adapted from the work of Fryback and Thornbury (9) and others.

$\mathrm{ROC}=$ receiver operating characteristic curve.
}

applications related to cancer. Referring physicians completed surveys indicating their intended management plan before PET and again after the results of the PET became available. The referring physicians indicated a change in intended management after PET from nontreatment to treatment or vice versa in over $36 \%$ of subjects (7). Citing a relationship between the institution of appropriate antitumor therapy and improved health outcomes, researchers working with CMS agreed that the observed change in treatment was a valid intermediate marker for improved health outcomes.

The November 2012 conference participants discussed 2 major concerns with using change in therapeutic intent as a surrogate marker for improved health outcomes for future evaluations of new PET radiopharmaceuticals or new indications of existing agents:

- The NOPR evaluated intended management rather than actual management. The difference between what is intended and what is actually done, often influenced by patient choice, can be considerable.

- The NOPR measured change in intended management after PET. If the change was inappropriate because the PET results were inaccurate (false positive or false negative), the NOPR would have overestimated the benefit of PET.

There is a robust selection of potentially clinically applicable PET radiopharmaceuticals in the pipeline. As evidence, Lalitha Shankar, MD, PhD, representing the Cancer Imaging Program (CIP) of the National Cancer Institute, reported that CIP currently is investigating a variety of PET radiopharmaceuticals that address specific molecular targets or measure biologic processes or phenomena, such as cell proliferation, angiogenesis, and hypoxia. CIP is developing an infrastructure to support the

\footnotetext{
${ }^{2}$ Conference attendees heard presentations addressing PET radiopharmaceuticals intended for application in oncology (Barry Siegel, MD; Bruce Hillner, MD), neurology (Peter Herscovitch, MD), and cardiology (Gary Heller, MD, PhD).
}

translation of innovative PET radiopharmaceuticals into clinical application.

The broad spectrum of agents under development suggests that the trial design and endpoints must depend, at least in part, on the specific characteristics of a new agent. However, there is one important commonality. Regardless of the application or target organ, ${ }^{2}$ at a minimum, CMS will likely require researchers to employ a defensible intermediate endpoint, such as change in therapy, to demonstrate that the use of the technology is "reasonable and necessary."

Although CMS cannot statutorily consider the costliness of a novel technology in its coverage deliberations, it is hard to imagine how, in this time of concern over health care expenditures, the potential cost to the Medicare program of covering a technology could be ignored completely. Stressing 3 key points relevant to the economics of an innovation, Craig Hunter, MPP, argued that value should be the final consideration for new PET radiopharmaceuticals. He went on to elucidate that

- Value includes both economic and noneconomic considerations;

- Economics becomes the method by which multiple value inputs are summarized into budget-comparable bases for decision making; and

- Establishing clear methods for evaluating economic endpoints prior to initiation of health technology assessment maximizes transparency and mitigates the risk of ineffective analyses.

Despite their importance, economic evaluations of innovative radiopharmaceuticals have not been uniformly applied. Perhaps this oversight is attributable to the risk of ignoring confounding factors that could lead to an incorrect result. As Hunter warned, the real risk is conducting a study that asks the wrong questions.

\section{CONSENSUS OF THE PARTICIPANTS}

The goals of the conference participants were to synthesize the presented information, add their own knowledge and perspectives, and generate a coherent structure for how future coverage evaluations of new PET radiopharmaceuticals should be designed and conducted. Recognizing there might be differences in preferred designs among different medical specialties, the participants were split into 3 groups-oncology, cardiology, and neurology.

\section{Considerations of the Oncology Group}

The oncology group identified the need for different endpoints for different possible uses of PET radiopharmaceuticals. The applications of greatest interest included staging, restaging, and detection of suspected recurrence; surveillance and diagnosis; monitoring response to therapy; and biological characterization.

The participants felt that PET plays an important role in the staging and evaluation of recurrence of cancer. They proposed a 3stage approach to this application, relying on observational studies without the need for randomized clinical trials:

- Stage I: Methodologically rigorous comparisons of the novel radiopharmaceutical with existing tests. All discrepant results should be subjected to histological verification. If the new test shows promise, it should progress to stage II. 
- Stage II: The establishment of a registry, addressing a sizable population of patients with cancer, the purpose of which would be to evaluate the frequency of change of intended therapy based on the conduct of the new test. Such a registry would be agreed upon in scope, length of time, and other significant parameters in order for CMS (or any payer) to be satisfied that the data collected would answer the coverage questions posed within a reasonable timeframe and cost.

- Stage III: Should stage II demonstrate a sizable effect of the new test in changing intended treatment, the group recommended that there be subgroup analyses to verify both that the change in treatment actually occurred and that the change in treatment was likely to be beneficial to the patient.

The oncology group felt that a particularly important application likely to be addressed by the development of new PET radiopharmaceuticals is monitoring the effects of treatment. Improved monitoring afforded by PET technology would allow ineffective treatment to be discontinued earlier and, more accurately, avoid overtreatment, thereby saving both cost to the health care system and harm to patients. The group considered the potential benefit of using PET to characterize tumor biology. The goal would be to image the expression of a therapeutic target or resistance factor and assess the temporal and spatial heterogeneity of that expression to guide treatment. Again, it was felt that randomized trials would not be necessary for this application. The desired goals should be achieved by correlating PET findings with an established standard of evidence, such as histopathology.

Finally, the oncology group indicated that PET may serve an important role by informing patients of their prognosis even in situations where there is no therapeutic alternative to address their condition, thereby empowering patients to avoid the adverse effects of treatment that would be futile while also saving the financial costs to themselves and payers.

\section{Considerations of the Cardiology Group}

The cardiology group considered both endpoints and context of future cardiac PET radiopharmaceuticals. The preferred primary endpoint for these innovations should be the impact of the test on changing the treating physician's treatment plans. Secondary endpoints might include the frequency and intensity of downstream diagnostic testing. Lesser, but possibly important, endpoints that might be useful for coverage decisions include improved patient access to treatment; a higher level of diagnostic accuracy; quantitative measures of function; better image quality; prognostic accuracy; and greater practitioner confidence in diagnosis and treatment. The application of such endpoints to clinical care may add value by allowing more accurate assignment of patients to low-risk or highrisk groups, thus affecting the intensity of their surveillance or treatment and increasing compliance with risk-modification regimens.

Finally, the group felt that CMS' emphasis on the impact of positive results in their coverage deliberations should be better balanced by recognition of the value of negative results. The group argued that if negative results compel a physician not to treat, or change a treatment decision, these results provide significant incremental benefit in the same manner as a positive result.

\section{Considerations of the Neurology Group}

The neurology group focused on expectations for the emergence of additional imaging agents directed at Alzheimer's disease. Like the oncology and cardiology groups, this group believed that, in most cases, a change in management leading to an incremental benefit to patients should be sufficient to support Medicare cover- age. Included among these benefits would be the more appropriate starting, not starting, or stopping of treatment resulting in one or more of the following: (1) significant symptom improvement allowing better function and cognitive performance; (2) the avoidance of toxic side effects attributable to the inappropriate use of anticholinesterase medication; or (3) improved quality of life, including access to available treatments, addressing safety issues, and personal and social planning earlier in the disease process, all of which are supported by the "value of knowing (8)."

An additional benefit would be reduced need for additional testing and burdensome medical visits as a result of using PET. Such an endpoint should be considered for inclusion in the design of future PET research.

With regard to this last item, neurology group members agreed with the cardiology group that there was significant value to knowing even when there is no available treatment option. Knowing is critical to patients and their families so that affected individuals have time to put their affairs in order before progression occurs.

\section{CONCLUSIONS}

Despite the great breadth of potential applications and affected organ systems that might be addressed by future PET radiopharmaceuticals and the diversity of participants, there was convergence of opinion as to what research designs and endpoints were most appropriate. Participants agreed that randomized controlled clinical trials are rarely necessary and that the use of intermediate endpoints, rather than the direct measurement of health outcomes, is the most appropriate approach to generate the data necessary to underpin CMS coverage deliberations. As the convener of the conference, MITA intends to use the advice proffered by the participants in developing future proposals to CMS.

\section{TAKE-HOME POINTS}

- There is a robust selection of new and potentially valuable PET radiopharmaceuticals in the pipeline poised for translation into clinical trials and eventually clinical practice.

- CMS coverage of any new technology is essential if the innovation is to become appropriately adopted into clinical practice so patients will have access when needed.

- CMS' benchmark for coverage of "reasonable and necessary" is that the technology provides a benefit to patients' health.

- With respect to diagnostic technologies, it is often difficult to parse the specific benefit derived from the test from the chain of diagnostic and therapeutic maneuvers that comprise the totality of clinical care. Diagnostic technologies should be considered differently than therapeutic interventions because of the incremental benefit the diagnostics provide in therapeutic decision making.

- Coverage of new PET radiopharmaceuticals should depend on clinical evidence of effect on intermediate endpoints, such as a beneficial change in clinical management (ie, change in subsequent therapeutic or diagnostic interventions) that can be linked to improved health outcomes.

- The outcomes, or endpoints, appropriate to assessing whether diagnostic interventions are reasonable and necessary are best characterized as "change in clinical management." This is distinct from the outcomes, or endpoints, classically applied in assessing whether therapeutic interventions are reasonable and necessary. 
Conference Participants

\begin{tabular}{|c|c|}
\hline Name & Organization \\
\hline Brian C. Abraham, MPA & MITA \\
\hline Zaver Bhujwalla, MD & $\begin{array}{l}\text { Johns Hopkins } \\
\text { University }\end{array}$ \\
\hline Linda B. Bresolin, PhD & RSNA \\
\hline Bonnie Clarke & SNNMI CTN \\
\hline Ward Digby, $\mathrm{PhD}$ & Siemens \\
\hline Frederic H. Fahey, DSc & SNMMI \\
\hline Richard A. Frank, MD, PhD & $\begin{array}{l}\text { Siemens Medical } \\
\text { Solutions }\end{array}$ \\
\hline Constantine Gatsonis, PhD & Brown University \\
\hline Janet Gelbach & Jubilant Draximage \\
\hline James Gilroy & Lilly \\
\hline Mickey Guiberteau, MD & RSNA \\
\hline Rosemarie Hakim, PhD & CMS \\
\hline Gary V. Heller, MD, PhD & $\begin{array}{l}\text { Intersocietal } \\
\text { Accreditation } \\
\text { Commission }\end{array}$ \\
\hline Peter Herscovitch, MD, FACP & $\mathrm{NIH}$ \\
\hline Bruce J. Hillman, MD & ACR Image Metrix \\
\hline Bruce E. Hillner, MD & $\begin{array}{l}\text { Va Commonwealth } \\
\text { University }\end{array}$ \\
\hline Marybeth Howlett, MEM & $\begin{array}{l}\text { Avid } \\
\text { Radiopharmaceuticals }\end{array}$ \\
\hline Craig A. Hunter, MPP & Lilly \\
\hline Louis B. Jacques, MD & CMS \\
\hline William Lawrence, MD & $\mathrm{AHRQ}$ \\
\hline $\begin{array}{l}\text { Howard C. Lewin, MD, FACC, } \\
\text { FASNC }\end{array}$ & ASNC \\
\hline Misty Long, RT (R)(N) & PETNET Solutions \\
\hline Jane Majcher & GE Healthcare \\
\hline Anita McGlothlin & ACR \\
\hline Andrew McKinley & ASNC \\
\hline Al Naqvi & WMIS \\
\hline Doug Neale & $\begin{array}{l}\text { Lantheus Medical } \\
\text { Imaging }\end{array}$ \\
\hline Virginia Pappas, CAE & CEO, SNMMI \\
\hline David Pendleton & $\begin{array}{l}\text { Navidea } \\
\text { Biopharmaceuticals }\end{array}$ \\
\hline Bruce Quinn, MD & Foley Hoag, LLP \\
\hline Dwaine Rieves, MD & US FDA \\
\hline Gail Rodriguez, PhD & MITA \\
\hline George Segall, MD & SNMMI \\
\hline Lalitha K. Shankar, MD, PhD & $\mathrm{NCl}$ \\
\hline Barry A. Siegel, MD & $\begin{array}{l}\text { Wash. U. School of } \\
\text { Medicine }\end{array}$ \\
\hline Wally Smith & $\begin{array}{l}\text { Lantheus Medical } \\
\text { Imaging }\end{array}$ \\
\hline Tamara Syrek Jensen, JD & CMS \\
\hline Karen M. Tripoli, RT (R)(N) & PETNET Solutions \\
\hline Randy VanCoughnett & $\begin{array}{l}\text { Lantheus Medical } \\
\text { Imaging }\end{array}$ \\
\hline Wolfgang A. Weber, MD & University of Freiburg \\
\hline Terri Wilson, MBA, CNMT & Cardinal Health \\
\hline Annemarie Wouters, PhD & Manatt Health Solutions \\
\hline Robert Zeman, MD & ACR \\
\hline
\end{tabular}

1. Hillman BJ, Frank RA, Rodriguez GM. New pathways to Medicare coverage for innovative PET radiopharmaceuticals: report of a Medical Imaging \& Technology Alliance (MITA) workshop. J Amer Coll Rad 2012;9:108-14.

2. Proposed decision memo http://www.cms.gov/medicare-coverage-database/details/ nca-decision-memo.aspx? NCAId=261 Accessed Nov. 29, 2012

3. Hillner BE, Siegel BA, Shields AF, et al. The impact of positron emission tomography (PET) on expected management during cancer treatment: findings of the National Oncologic PET Registry. Cancer 2009;115:410-8.

4. Hillner BE, Siegel BA, Shields AF, et al. Relationship between cancer type and impact of PET and PET/CT on intended management: findings of the National Oncologic PET Registry. J Nucl Med 2008;49:1928-35.

5. Hillner BE, Siegel BA, Liu D, et al. Impact of positron emission tomography/ computed tomography and positron emission tomography (PET) alone on expected management of patients with cancer: initial results from the National Oncologic PET Registry. J Clin Oncol 2008;26:2155-61.

6. Hillner BE, Siegel BA, Shields AF, et al. Impact of dedicated brain PET on intended patient management in participants of the national oncologic PET Registry. Mol Imaging Biol 2011;13:161-5.

7. Hillner BE, Siegel BA, Hanna L, et al. Impact of 18F-FDG PET used after initial treatment of cancer: comparison of the National Oncologic PET Registry 2006 and 2009 cohorts. J Nucl Med 2012;53:1-7.

8. Johnson KA, Minoshima S, et al. Appropriate use criteria for amyloid PET: a report of the Amyloid Imaging Task Force, the Society of Nuclear Medicine and Molecular Imaging, and the Alzheimer's Association. J Nucl Med 2013;54:476-90.

9. Fryback DG, Thornbury JR. The efficacy of diagnostic imaging. Med Decis Making 1991;11:88-94. 